\title{
15. Combined Orbitofrontal Craniotomy
}

\author{
Hiroshi Matsumura and Yasumasa Makita \\ Neurosurgical Department of Tenri Hospital
}

Joji Handa, Toru Watanabe and Toshimichi Fukuda

Neurosurgical Department of Kyoto University Medical School

Tym, R. (1961) orginated the orbitofrontal craniotomy to remove 10 piloid gliomas of the optic tracts. This procedure was modified in our clinic and used in following 2 operations.

1) Sphenoidal ridge meningioma wiwth intensive exophthalmos: Orbitofrontal craniotomy with large frontal bone flap continuing to the subtemporal decompression behind was performed. Tumor extended from middle fossa into the orbital cavity lateral and over the orbital content. All tumor was removed except for tiny tumor itssue attaching to the carotid wall. Eye ball was successfully reposited into the orbital cavity.

2) Constriction of the optic canal due to fibrous dysplasia with slight exophthalmos: Optic atrophy was already perfect but we were obliged to operate by the supplication of family. Orbitofrontal craniotomy with small frontal bone flap was performed. Whole optic canal and superior orbital fissure were decompressed by the manipulation from anterior fossa. Sight was not regained.

Wide operative field to the skull base and orbit was obtained during surgery. Extensive elevation of the brain from the skull base was unnecessary in this operation. Postoperative course of both cases were uneventful.

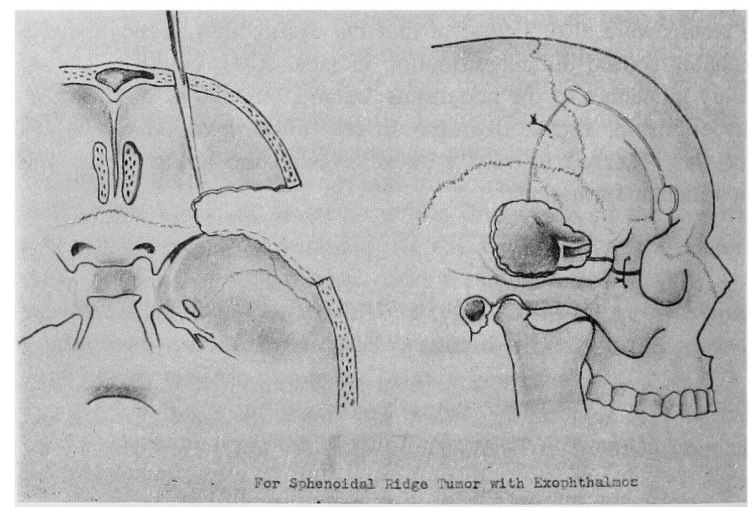

Fig. 1. 


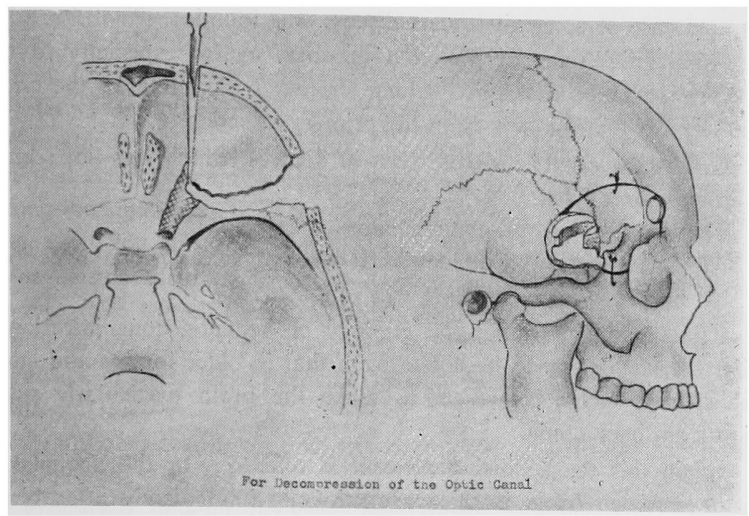

Fig. 2.

\title{
16. Surgical Hematogenous Pyogenic Infection of the Brain in Infants and its Etiology
}

\author{
Shi Hui Huang, Akira Hakuba and Atsuo OKazaKI \\ Yodogawa Christian Hospital, Osaka
}

From 1960 through 1965, 15 cases of pyogenic infection of the brain in infants under 4 months of age were treated. Among them 10 cases were suspected to be hematogenous: 6 brain abscesses, 3 ventricular empyemas and 1 purulent meningitis with hydrocephalus. Bacterically we found gram (-) organisms in 4 cases, staphylococcus in 2 cases, no growth in 1 case, mixed infection with gram $(-)$ bacillus and gram $(+)$ coccus in case and unknown type in two cases.

A history of difficult delivery was found in 8 cases. Among them 1 case got history of difficulty by mother but not from the physician. Neonatal difficulties were seen in all 10 cases. The type of difficult delivery was induction and stimulation, prolongegd membrane rupture, instrumental delivery, and others mean that the breach pressentation, prolonged laber for 3.5 days and cesarian section. Difficulties in neonatal period was prematurity, asphyxia, fever and vomiting, convulsion and injection at brith or repeated injections within 10 days of life.

From the 10 cases of hematogenous infection, the following gfindings can be summarized: 